\title{
Left ventricular assessment using real time three dimensional echocardiography
}

\author{
L Sugeng, L Weinert, R M Lang
}

Heart 2003;89(Suppl III):iii29-iii36

$\mathrm{T}$ he ability to accurately assess left ventricular function non-invasively is essential for patient management since it has been known for years that left ventricular ejection fraction serves as an important predictor of long term survival. Although two dimensional (2D) echocardiography is routinely used in clinical practice to obtain information on left ventricular dimension, wall thickness, and function, this technique is limited because it relies heavily on geometrical assumptions to provide quantitative parameters of left ventricular function. In order to avoid these geometrical constraints, three dimensional (3D) reconstructions of the left ventricular cavity have been performed over the last 30 years using a variety of method.

Although multiple studies have validated the superiority of 3D over 2D echocardiography to assess left ventricular function, 3D methods have not been embraced in clinical practice because of the cumbersome methodology used to date for both data acquisition and analysis. This manuscript will review prior 3D techniques used to quantitate left ventricular function and present an overview of the recently introduced matrix array transducer capable of generating real-time 3D transthoracic echocardiographic images.

\section{D ECHOCARDIOGRAPHIC METHODS}

Estimations of left ventricular volumes were first performed using $\mathrm{M}$ mode echocardiography. Published data using this unidimensional technique reported a large volume discrepancy between this methodology and angiographic data. Although 2D echocardiography has improved left ventricular volume calculations, underestimations are still frequently reported when compared with gold standard methods such as angiography and magnetic resonance imaging (MRI). ${ }^{1-4}$

Three separate steps are required to assess left ventricular function using 3D echocardiography: (1) data acquisition, (2) image processing, and (3) data analysis. Acquisition of a 3D volume dataset may be obtained with a variety of methods such as free-hand scanning using mechanical or nonmechanical locators, gated sequential acquisition methods, and sparse and full matrix array transducers capable of obtaining real time data sets. In the next section, the strengths and limitations of these methods will be briefly discussed.

\section{Free-hand scanning method}

Initial efforts to reconstruct a stationary left ventricular cast from which left ventricular volumes could be derived, used a mechanical free-hand scanning method. Five short axis images were acquired and aligned along a long axis which served as the reference image. ${ }^{5-7}$ To estimate left ventricular volumes, endocardial borders of all end systolic and end diastolic frames had to be traced manually. Shortly thereafter, investigators used a non-mechanical position tracking system employing either an acoustic locator or spark gap. Compared to the mechanical free-hand scanning method, this method allowed more freedom of movement for the operator resulting in improved image quality. ${ }^{8-11}$ With this methodology, investigators acquired six to eight non-parallel, equally spaced, non-intersecting short axis planes which were also aligned along the left ventricle obtained from the parasternal long axis view, which served as the reference image.

Image processing and data analysis were performed offline. Surface reconstructions of the left ventricle were done using a ray tracing technique wherein measurements of left ventricular volumes were derived from the summation of tetrahedrons. This method also required off-line tracing of endocardial borders from end diastolic and end systolic frames. Estimated left ventricular volumes, masses and ejection fractions obtained using the free-hand method were significantly more accurate and had less variability compared to the 2D biplane summation of disks method, when using MRI and/or radionuclide angiography as the gold standard for comparison. ${ }^{9} 10$ 12-17

Non-mechanical devices such as spatial acoustic locators and spark gap systems, which are attached to commercially available transducers, allowed the sonographer to have increased freedom of movement with the transducer resulting in easier and improved data acquisition. However, both of these methods require bulky instrumentation, which limits their portability. This limitation was compounded by the interference created by lines, cables or metal hospital beds. Furthermore, with both these methods, left ventricular volume data sets are obtained over multiple cardiac cycles, which were usually acquired gated to ECG and respiration. Cardiac translation secondary to respiration or patient movement may also contribute to the acquisition of poor data quality, which may result in inaccurate volume calculations.

\section{Gated sequential scanning method}

This mode of data acquisition collects 2D images sequentially, either in a rotational, fan-like or parallel manner from one acoustic window, gated to ECG and respiration. The most frequently used method is the rotational approach in which images are acquired using a multiplane transoesophageal probe. ${ }^{18-20}$ Alternatively, a transthoracic approach may be used with an external stepper motorised device attached to a commercially available transducer, while the ultrasound unit is interfaced with a dedicated computer system (Echoscan, Tomtec, GMBH, Munich, Germany). ${ }^{21}$ Images are collected over $180^{\circ}$ rotation with intervals of $2-5^{\circ}$ using ECG and respiratory gating.

Once acquired, the images are post-processed off-line on a dedicated computer system and converted to a Cartesian coordinate system to obtain a conical volume data set. From this dataset, any desired cut plane can be derived and structures of interest rendered, with the operator determining threshold and opacity values. Additional smoothing algorithms may be subsequently applied to reduce noise and spatial artefacts. This mode of acquisition resulting in 
volume rendered images has the advantage of providing extensive surface detail of cardiac anatomy. The spatial relations between different cardiac structures can be appreciated in detail, which is of benefit when evaluating patients with complex congenital abnormalities ${ }^{21-24}$ or valvar disease. ${ }^{18} 2025$

Assessment of left ventricular function is performed offline, using either a disc summation method or a longitudinal semi-automated method. Validation of left ventricular volumes, mass, and ejection fraction using the gated sequential acquisition method has been performed against different gold standards, such as radionuclide angiography (RNA) and MRI. ${ }^{26-33}$ Unfortunately, the prolonged acquisition times have hindered the routine clinical use of this method. To reduce the time of data collection, investigators increased the rotation acquisition interval reporting that accurate and reproducible left ventricular volumes and ejection fractions can still be achieved. ${ }^{34}{ }^{35}$ The gated sequential acquisition method has been successfully used in irregularly shaped hearts resulting in accurate measurements of left ventricular volumes and ejection fractions, in both in vitro and in vivo studies..$^{27} 36$ The gated sequential acquisition method has also been reported to accurately measure left ventricular mass from both the transthoracic and transoesophageal approach. ${ }^{37} 38$ This methodology has also been used to estimate the myocardium at risk during experimental acute myocardial infarctions, as well as the residual infarct volume and mass after revascularisation. ${ }^{30}{ }^{39-41}$

More recently, free-hand transthoracic gated sequential scanning has been performed using a magnetic tracking device attached to a commercially available ultrasound transducer. ${ }^{42-44}$ Data are acquired by manually moving the transducer in small increments in a fan-like manner with ECG and respiratory gating to obtain a pyramidal volume data set. Rendering of $3 \mathrm{D}$ images is then performed in a manner similar to the method above described. Free-hand scanning is particularly useful for visualising fine details of the mitral valve apparatus and to accurately measure mitral valve orifice areas. The main advantage of this method is that the sonographer is in complete control of the transducer's position thereby allowing rapid data acquisition from the best acoustic window.

\section{Real time 3D}

Routine clinical use of 3D echocardiography has been hindered by the prolonged and tedious nature of data acquisition. Image processing is not only time consuming but also requires dedicated manpower to generate 3D reconstructions and useful quantitative data. Accordingly, the recent introduction of real time 3D echocardiographic imaging techniques is of great interest since this methodology may circumvent many of the above mentioned limitations.

\section{Sparse array transducer}

Real time 3D imaging was initially performed using a sparse array matrix transducer (2.5 or $3.5 \mathrm{MHz}$ ), which consisted of 256 non-simultaneously firing elements. This transducer acquired a pyramidal volume data set measuring $60^{\circ} \times 60^{\circ}$ within a single heart beat. Echocardiographic images were then displayed "on line" using simultaneous orthogonal (B scan images) as well as 2-3 parallel short axis planes (C scans)..$^{45} 46$

Similar to other 3D methods, the sparse array transducer resulted in accurate left ventricular volumes, ejection fraction, and mass when compared to gold standard techniques, such as MRI and RNA. ${ }^{47-51}$ The sparse array transducer was also used advantageously during stress testing, because all post-exercise images were simultaneously acquired during a
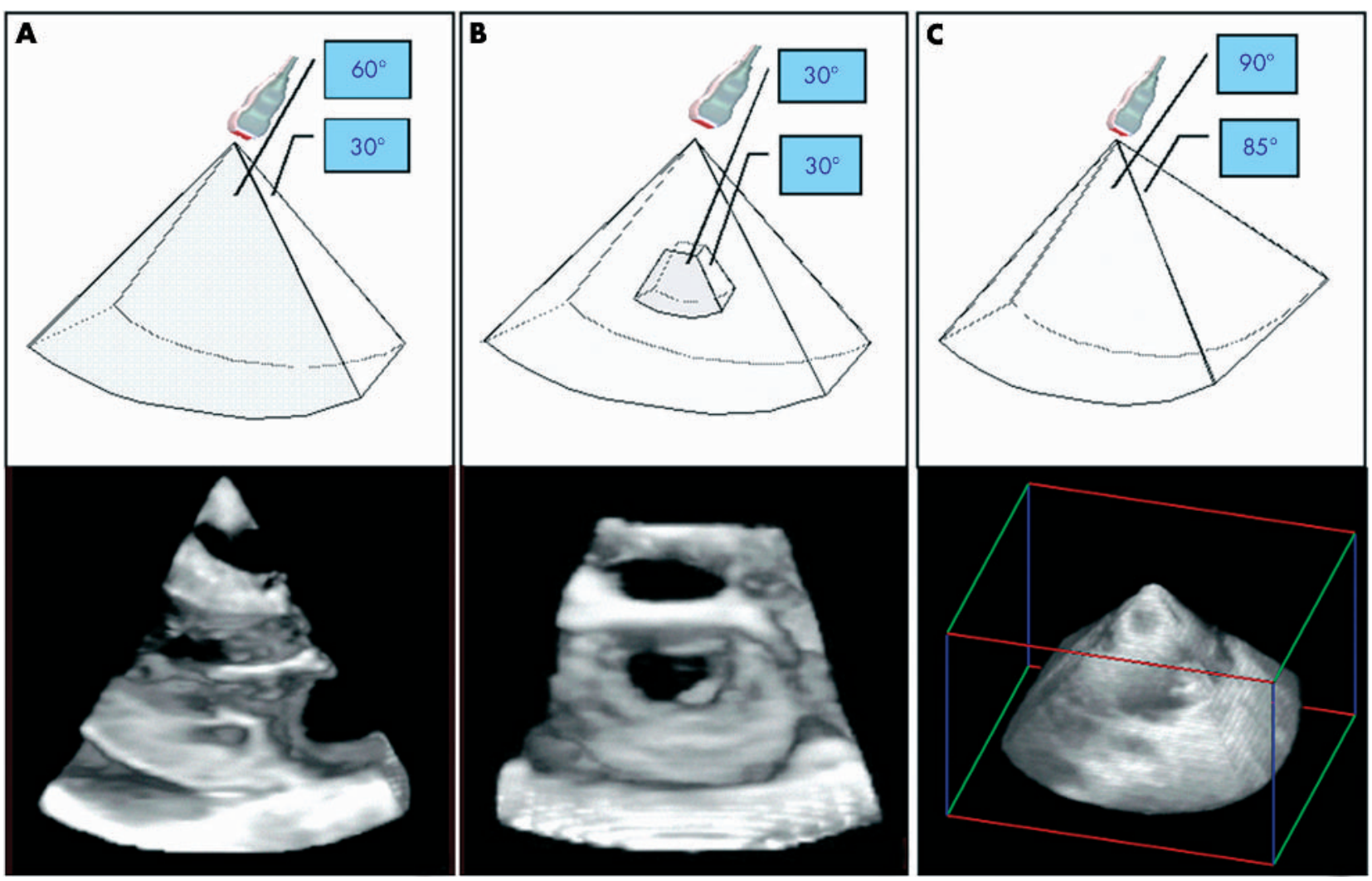

Figure 1 Different modes of data acquisition using the matrix array transducer: (A) narrow angled scan, (B) zoom mode, and (C) wide angled acquisition. See text for further explanation. 
single heart beat which was at higher peak stress heart rates compared to conventional stress tests. ${ }^{52} 53$

Although 3D real time images were acquired using this novel transducer, it continued to have several disadvantages that precluded its routine clinical use. The ultrasound images were of relatively poor quality, frame rates were low, and the pyramidal volume had a relatively narrow sector angle of $60^{\circ}$, which resulted in the inability to accommodate larger ventricles. Moreover, the images obtained with this system were not volume rendered on-line; instead, they consisted of computer generated 2D cut planes derived from the 3D volume dataset.

\section{Full matrix array}

Recently, a full matrix array transducer (X4, Phillips Medical Systems, Andover, Massachusetts, USA), which utilises 3000 elements, in contrast to the 256 elements of the sparse matrix array probe, has been developed. This new development in transducer technology has resulted in (1) improved side-lobe performance (contrast resolution), (2) higher sensitivity and penetration, and (3) harmonic capabilities which may be used for both grey scale and contrast imaging. In addition, this transducer displays "on-line" 3D volume rendered images and is also capable of displaying two simultaneous orthogonal 2D imaging planes (that is, biplane imaging).
Real time volume rendered 3D imaging

The full matrix array transducer has several modes of data acquisition (fig 1): (A) narrow angle acquisition which consists of $60^{\circ} \times 30^{\circ}$ pyramidal volumes displayed in a volume rendered manner in real time without the need for respiratory gating; (2) the "zoom mode" which allows a magnified view of a subsection of the pyramidal volume $\left(30^{\circ} \times 30^{\circ}\right.$ sector in high resolution); and lastly (3) wide angled acquisition which is used to collect the entire left ventricular volume. In this acquisition mode, four wedges $\left(15^{\circ} \times 60^{\circ}\right)$ are obtained over eight consecutive cardiac cycles during a breath hold with ECG gating. The first two modes of data acquisition are predominantly used to visualise cardiac and valvar morphology. Images of heart valves acquired in this manner provide unique views, which are not always readily obtainable using conventional 2D echocardiography.

In contrast, the wide angled acquisition mode is often used to acquire the entire left ventricular volume in order to perform detailed analysis of global and regional wall motion. This matrix array is capable of acquiring data at three levels of image resolution during both narrow and wide angled acquisition modes, which in turn has an impact on the size of the pyramidal volume. Data are stored digitally on CD ROM and may be transferred to an off-line computer for quantitative purposes.

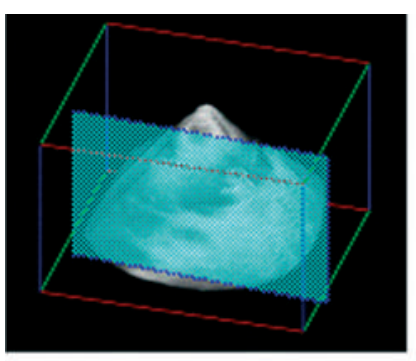

4 Chamber

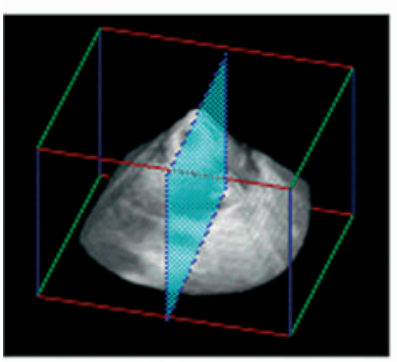

2 Chamber

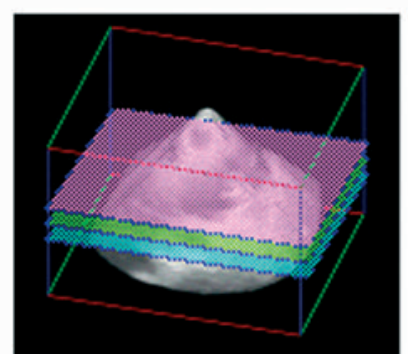

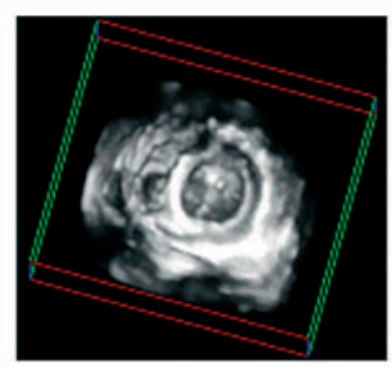

Base

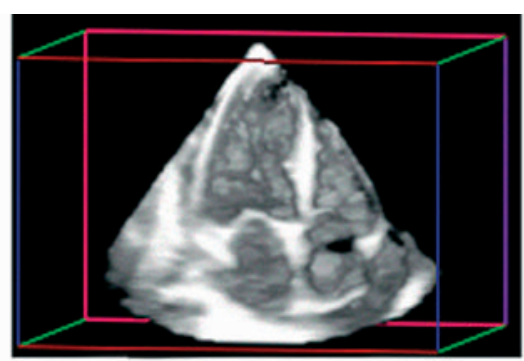

Anterior wall

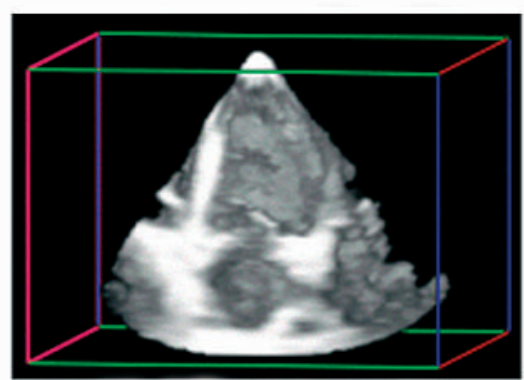

$\checkmark$ septal wall

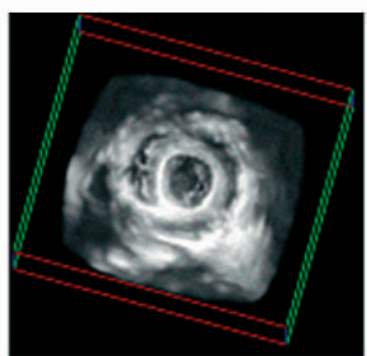

Mid

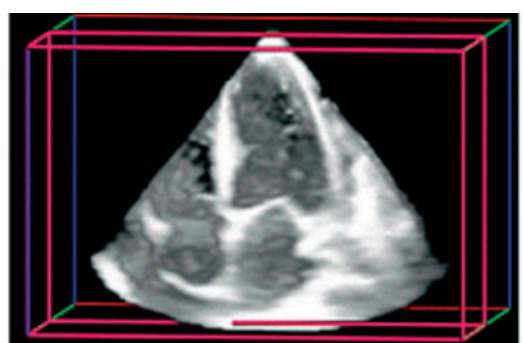

Posterior wall

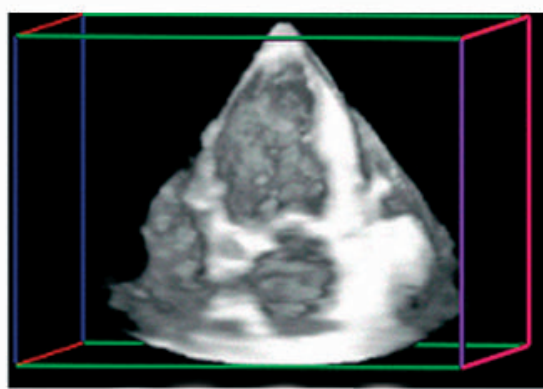

Lateral wall

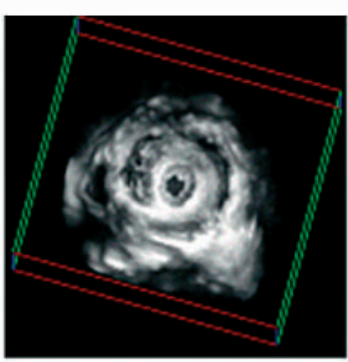

Apex

Figure 2 Wide angled scan of the left ventricle sliced using multiple cut planes. From a typical apical four chamber view using a longitudinal cut (top row), the septal and lateral walls are seen along with the surface of the anterior and posterior wall. From the two chamber view (middle row), the anterior and inferior walls are visualised together with the surface of the septal and lateral wall. Multiple cuts of the short axis from base to apex may be also derived from this scan, as seen on the bottom row. 
The left ventricle (apical four, three, and two chamber views) is usually acquired from the apical window using a wide angled acquisition (fig 2). Images may be displayed either using orthogonal long axis views, or using multiple short axis views, obtained at the level of the left ventricular apex, papillary muscles, and the base (fig 2, bottom row). For example, when slicing the heart orthogonally in a four chamber view, the septal and lateral walls together with the entire surface of the anterior or posterior wall are visualised (fig 2, top row). Likewise, in a two chamber view, the anterior and inferior walls are imaged in conjunction with the entire surface of the septum or lateral walls, depending upon the orthogonal cut used (fig 2, middle row). The right ventricle can also be assessed from a traditional four chamber.

\section{Left ventricular analysis}

In contrast to 2D echocardiography, 3D echocardiography does not rely on geometric assumptions, to calculate left ventricular volumes. This constitutes a real advantage in ventricles with odd shapes and wall motion abnormalities. ${ }^{16505455}$ Similarly, the unique geometrical shape of the right ventricle has precluded accurate quantification using traditional echocardiographic methods. Transthoracic 3D echocardiography has the potential of overcoming these limitations resulting in accurate measurements of right ventricular size and function. ${ }^{56-61}$
Multiple studies have found 3D calculated left ventricular volumes, ejection fractions, and left ventricular mass values to be comparable to those obtained with nuclear imaging and magnetic resonance imaging. However, since gated acquisition methods including free-hand scanning are tedious, time consuming, and relatively non-portable, calculations of left ventricular ejection fraction from 3D reconstructions have not been incorporated in the routine echocardiographic studies. Additional disadvantages of the previously used 3D methodologies include: (1) data sets are acquired over multiple heart cycles; (2) data processing was slow because of the limitations of computer technology; (3) calculation of left ventricular volumes was performed on an off-line system which requires tedious manual tracing of endocardial borders; and (4) the left ventricle was displayed using static wire frame which in addition failed to provide anatomical information.

Quantification of left ventricular volumes and mass using real time 3D echocardiography is usually performed from an apical wide angled acquisition using different methods. Currently, data analysis is performed on a desktop or laptop computer with dedicated 3D software (4D LV analysis, TomTec GMBH, Munich, Germany). Since a dataset comprises the entire left ventricular volume, multiple slices can be obtained from the base to the apex of the heart to evaluate wall motion. This acquisition can be combined with the use
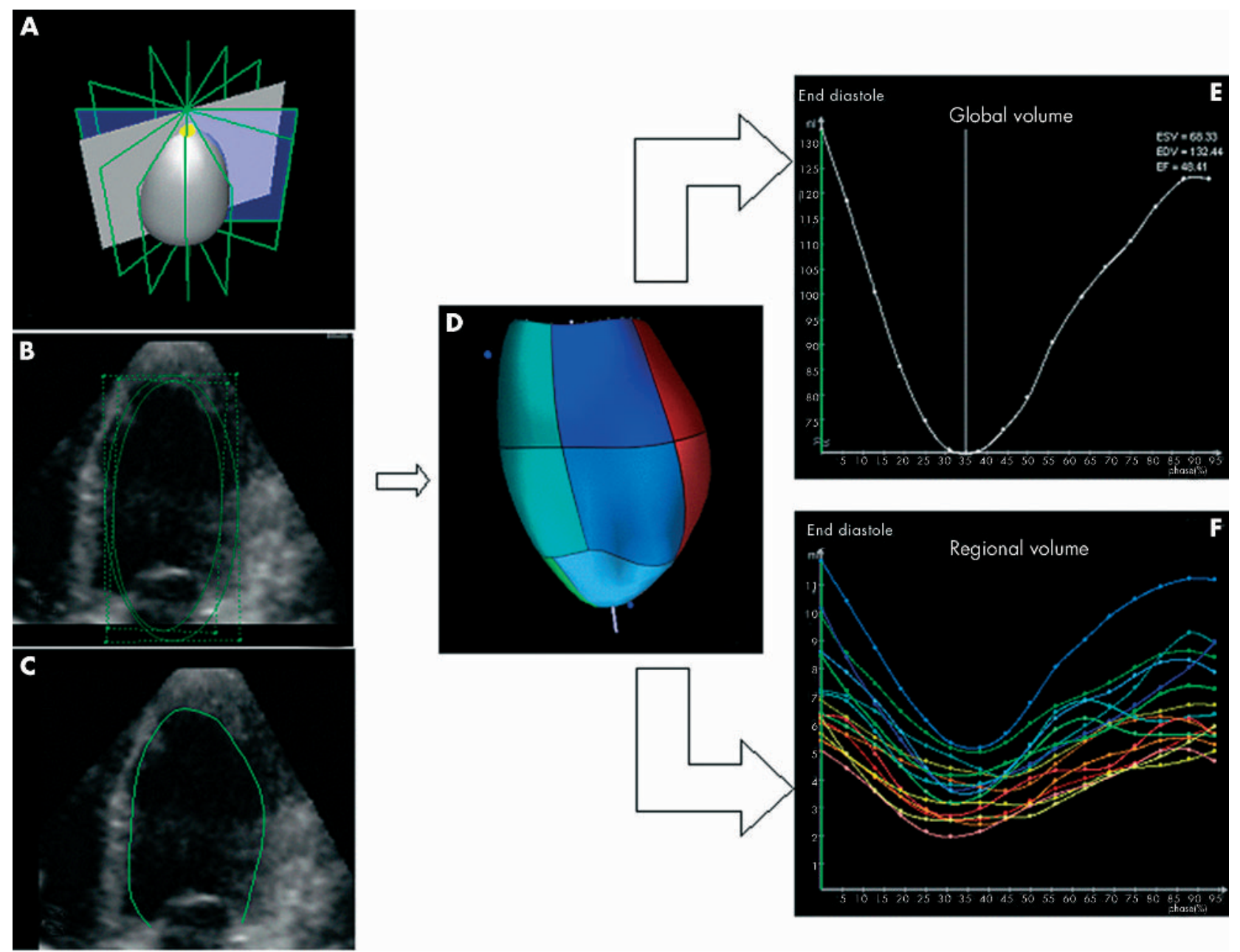

Figure 3 Panel A depicts the pyramidal volume of data divided automatically into eight equi-angled longitudinal slices through the apex. Panels B and $C$ depict the automated border detection algorithm used to track endocardial borders throughout the cardiac cycle. A dynamic ventricular cast is automatically displayed as a result of the endocardial borders and surface reconstruction (panel D). Global volumes and ejection fraction is displayed in panel E. Regional volumes of all 16 segments are shown in panel $F$. 

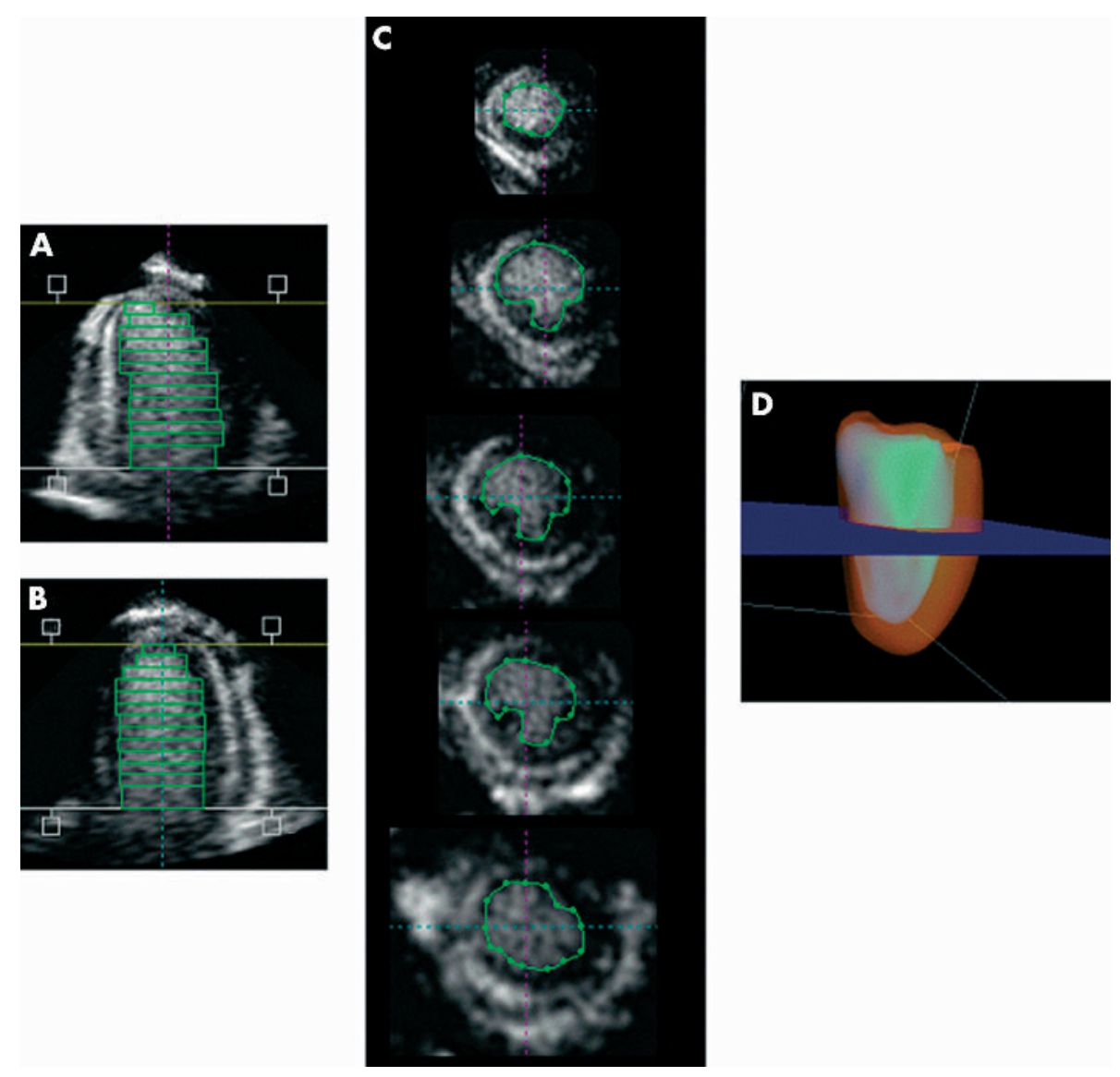

Figure 4 The disc summation method is an alternative method to calculate left ventricular volume and ejection fraction. The left ventricle is placed in a longitudinal position (panels A and B). With predefined distance intervals, multiple short axis cut planes are derived and endocardial borders traced in all end systolic and end diastolic frames (panel C). The summation of the volumes of each slice results in left ventricular volumes demonstrated in panel $D$.
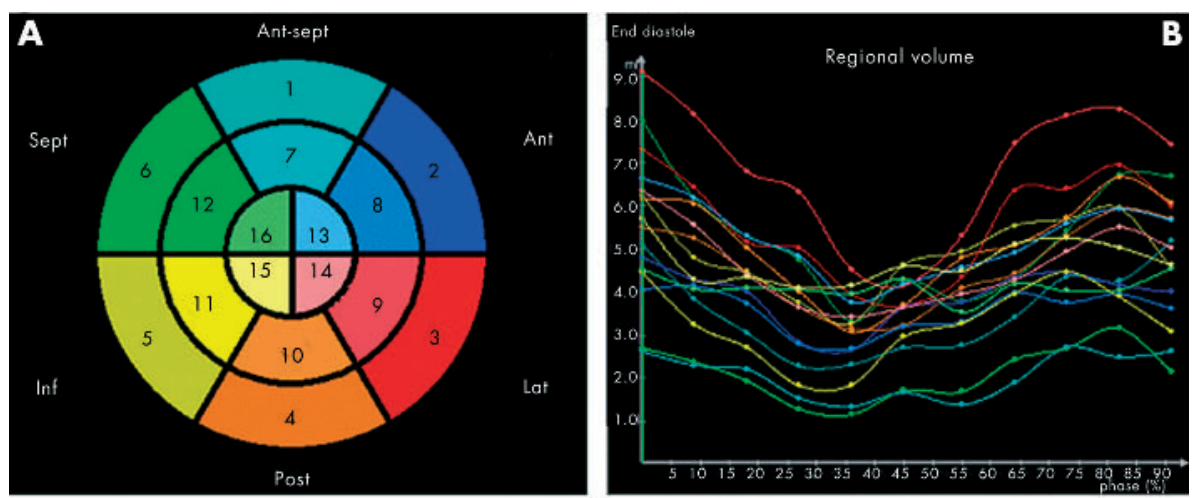

Figure 5 Bulls-eye demonstrating the left ventricle divided into 16 segments, corresponding to the regional volume of each segment (panel A). For each segment a regional volume curve is displayed (panel B). An example of a regional volume is depicted in panel $C$. Three dimensional echocardiography may help depict the more uniform mechanism of contraction during biventricular pacing. Preliminary observations demonstrate more synchronised regional volume curves, as seen in panel $D$, compared to panel $B$.
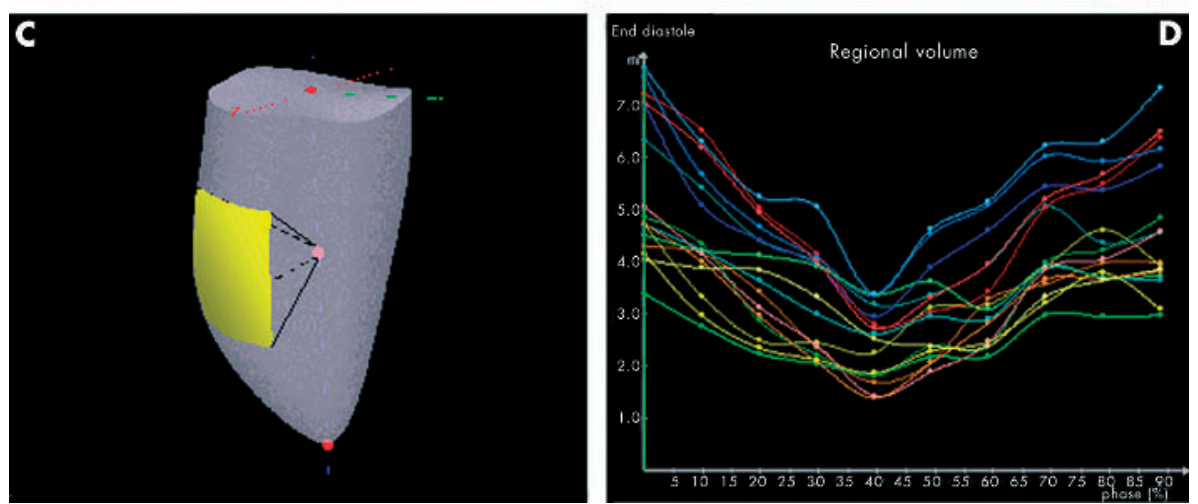

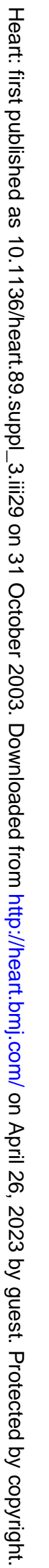




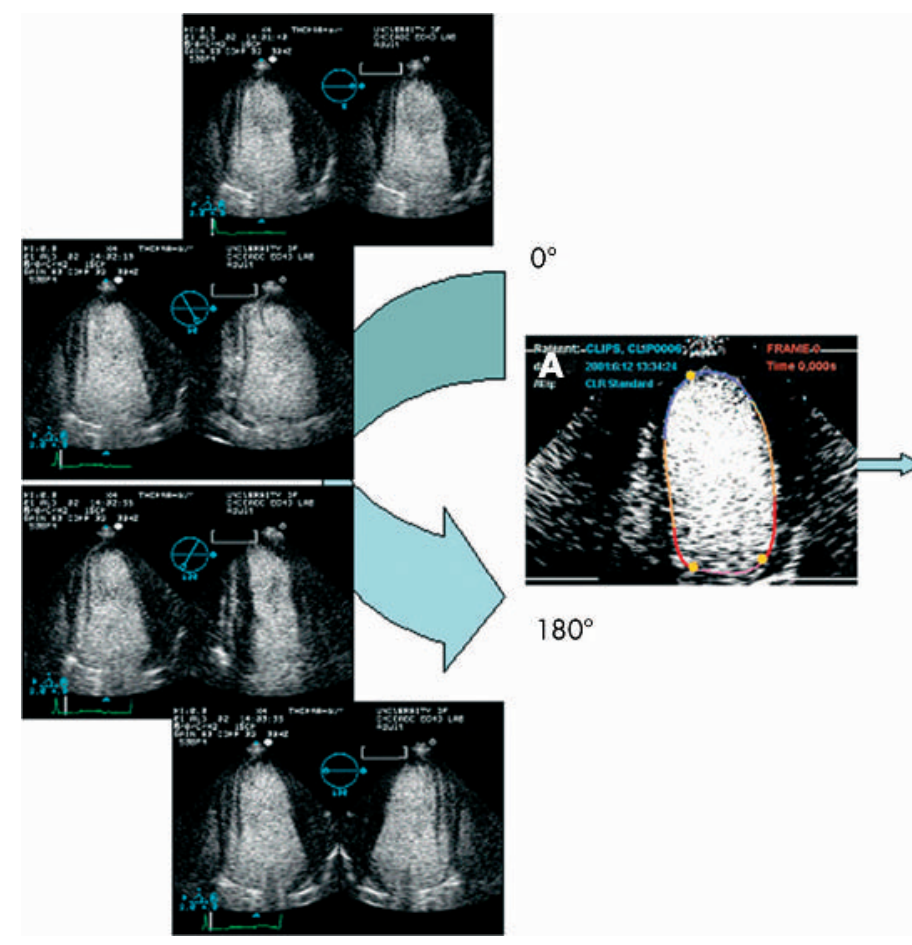

Figure 6 Biplane imaging from the apical windows provides an alternative method of data acquisition which may be useful in patients with dilated ventricles. Continuous contrast infusion enhances the endocardial border facilitating automated tracking (panel A). Multiple images are obtained at $10^{\circ}$

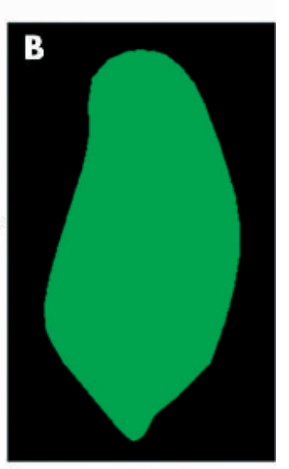
increments over $180^{\circ}$ rotation without respiratory gating shown on the left. A sphere is then fitted over the set of contours to calculate volume and ejection fraction (panel B).

of an infusion of contrast, particularly in patients with difficult acoustic window in whom it might be of benefit to improve the delineation of the endocardial border.

The $3 \mathrm{D}$ volume data set is then automatically divided into a number (that is, $\mathrm{n}=8$ ) of predetermined equi-angled longitudinal slices through the apex (fig 3, panel A). The midpoint of the mitral valve annulus, apex, and aortic valve are identified as landmarks. In each of the longitudinal slices an automated border detection algorithm is then used to track endocardial borders throughout the cardiac cycle (fig 3, panels B, C) to obtain a dynamic cast of the left ventricle, as well as instantaneous global and regional left ventricular volumes versus time curves (fig 3, panels D-F). This method of data analysis is semi-automated. In preliminary work, the volumes obtained with this method compare favourably with those obtained with cardiac MRI. An alternative method of calculating ventricular volumes from a real time 3D cardiac volume data set is using the disc summation method, which has been well validated in the past (fig 4). With this method, multiple short axis cut planes are obtained from base to apex using a predefined distance interval. In each short axis slice, endocardial borders at end systole and end diastole are traced and the summation of these volumes at end systole and end diastole are used to calculate left ventricular ejection fraction.
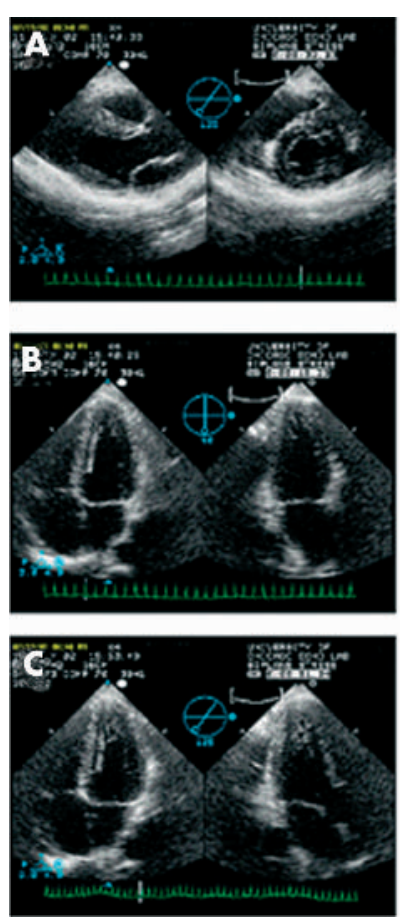

Figure 7 Comparison of the decline in heart rate at the time of data acquisition between the traditional $2 \mathrm{D}$ probe and the $\times 4$ transducer as demonstrated by the graph. ${ }^{63}$ Biplane views are displayed on the left in panels A-C.

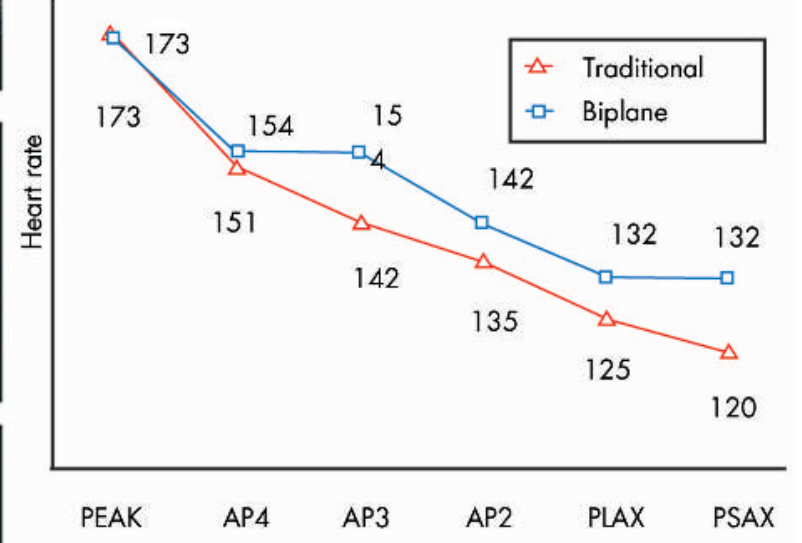


The combined use of an infusion of a contrast agent, such as Definity, during a wide angled acquisition can be used to improve endocardial border definition. Contrast echocardiography may result in more accurate quantification of left ventricular ejection fraction and myocardial mass particularly in patients with difficult acoustic windows. In order to obtain uniform contrast opacification, a continuous infusion of contrast is preferably used since the use of multiple scan lines destroys microbubbles at great speed. Multiple contrast enhanced slices of the left ventricle from base to apex can be obtained (fig 5). This acquisition mode may be also useful during dobutamine stress testing because the time required for data acquisition will be shortened substantially. Similarly, the ability to evaluate myocardial perfusion in three dimensions will provide unique information on the extent and location of perfusion defects.

Biventricular pacing in patients with severe left ventricular dysfunction has been shown to improve symptoms, but the mechanism responsible for this benefit is still the subject of controversy. Three dimensional echocardiography may be helpful to elucidate this mechanism because of its ability to clearly display changes in both global and regional volumes during different biventricular pacing settings. Preliminary observations demonstrate that regional contractility occurs during biventricular pacing in a more synchronised manner (fig 6).

\section{Biplane imaging}

Biplane imaging is a new imaging modality wherein the matrix transducer displays two orthogonal views in 2D simultaneously. The first imaging plane is used as the reference image, while the second plane may be rotated manually along the long axis of the left ventricle with increments of as small as $5^{\circ}$. Since two imaging planes are simultaneously displayed, this imaging modality will reduce the time required to complete a standard 2D echocardiographic examination. Additionally, using this transducer it is also possible to obtain unique $2 \mathrm{D}$ views such as the short axis of the tricuspid valve or orthogonal views of the pulmonary valve, which are not readily available from traditional acoustic windows using phased array transducers.

Biplane imaging can also be used as an alternative method to reconstruct the left ventricle. This technique may be particularly useful in patients with enlarged ventricles in which the wide angled scan may not be able to accommodate the entire left ventricle. A sequence of orthogonal images is obtained at predetermined angles (that is, $10^{\circ}$ over $180^{\circ}$ rotation) without respiratory gating. Using an automated tracking system, all contours of each scan plane are then converted to obtain a 3D volume dataset. A virtual sphere is fitted over the set of contours to calculate a left ventricular volume and ejection fraction (fig 6). ${ }^{62}$

We have demonstrated that the use of biplane imaging during exercise stress testing reduces the time required for data acquisition. This method has the potential to increase the diagnostic accuracy of treadmill stress echocardiography because of the possibility of acquiring peak stress images at higher heart rates. A study recently performed on 19 healthy subjects using biplane imaging during treadmill stress testing demonstrated a 10 (7) seconds reduction in the acquisition of all imaging planes with a mean (SD) acquisition time for biplane of 28 (7) seconds versus 38 (8) seconds for 2D. ${ }^{63}$ Since heart rate declines rapidly after cessation of exercise, the reduced acquisition time had a significant impact on the heart rates of the images recorded in each view (fig 7).

\section{FUTURE DIRECTIONS}

In the next few years, real time 3D echocardiography will surely become part of the routine echocardiographic examination. Future advancements in transducer and computer technology will allow wider angled acquisitions to be completed in a single cardiac cycle. Left ventricular quantification will be performed on the ultrasound unit thereby eliminating off-line analysis. This is crucial if the matrix array probe is to be used to guide interventional procedures in the cardiac catheterisation laboratory. In addition, better resolution will be required to maximise the diagnostic yield of this transducer, particularly in the far field. Also in the coming years, miniaturisation of the matrix array transducer technology will enable the acquisition of real time 3D transoesophageal images.

\section{Authors' affiliations}

L Sugeng, L Weinert, R M Lang, Section of Cardiology, Department of Medicine, University of Chicago Medical Center, Chicago, Illinois, USA

Correspondence to: Roberto M Lang, MD, University of Chicago, MC 5084, 5841 S. Maryland Avenue, Chicago, IL 60637, USA; rlang@medicine.bsd.uchicago.edu

\section{REFERENCES}

1 Starling MR, Crawford MH, Sorensen SG, et al. Comparative accuracy of apical biplane cross-sectional echocardiography and gated equilibrium radionuclide angiography for estimating left ventricular size and performance. Circulation 1981;63:1075-84.

2 Folland ED, Parisi AF, Moynihan PF, et al. Assessment of left ventricular ejection fraction and volumes by real-time, two-dimensional echocardiography. A comparison of cineangiographic and radionuclide techniques. Circulation 1979;60:760-6.

3 Erbel R, Schweizer P, Lambertz H, et al. Echoventriculography-a simultaneous analysis of two-dimensional echocardiography and cineventriculography. Circulation 1983;67:205-15.

4 Schnittger I, Fitzgerald PJ, Daughters GT, et al. Limitations of comparing left ventricular volumes by two dimensional echocardiography, myocardial markers and cineangiography. Am J Cardiol 1982;50:512-9.

5 Raichlen JS, Trivedi SS, Herman GT, et al. Dynamic three-dimensional reconstruction of the left ventricle from two-dimensional echocardiograms. J Am Coll Cardiol 1986;8:364-70.

6 Nixon JV, Saffer SI, Lipscomb K, et al. Three-dimensional echoventriculography. Am Heart J 1983;106:435-43.

7 Geiser EA, Lupkiewicz SM, Christie LG, et al. A framework for threedimensional time-varying reconstruction of the human left ventricle: sources of error and estimation of their magnitude. Comput Biomed Res 1980;13:225-41.

8 King DL, Harrison MR, King DL, et al. Improved reproducibility of left atrial and left-ventricular measurements by guided 3-dimensional echocardiography. J Am Coll Cardiol 1992;20:1238-45.

9 Gopal AS, King DL, Katz J, et al. Three-dimensional echocardiographic volume computation by polyhedral surface reconstruction: in vitro validation and comparison to magnetic resonance imaging. J Am Soc Echocardiogr 1992;5:115-24.

10 Sapin PM, Schroeder KD, Smith MD, et al. 3-Dimensional echocardiographic measurement of left-ventricular volume in-vitro-comparison with 2dimensional echocardiography and cineventriculography. J Am Coll Cardiol 1993;22:1530-7.

11 Handschumacher MD, Lethor JP, Siu SC, et al. A new integrated system for three-dimensional echocardiographic reconstruction: development and validation for ventricular volume with application in human subjects. J Am Coll Cardiol 1993;21:743-53.

12 Siu SC, Rivera JM, Guerrero JL, et al. 3-Dimensional echocardiography-invivo validation for left-ventricular volume and function. Circulation 1993;88:1715-23.

13 Gopal AS, Keller AM, Rigling R, et al. Left-ventricular volume and endocardial surface-area by 3-dimensional echocardiography-comparison with 2surface-area by 3-dimensional echocardiography-comparison with 2-
dimensional echocardiography and nuclear-magnetic-resonance imaging in normal subjects. J Am Coll Cardiol 1993;22:258-70.

14 Sapin PM, Schroder KM, Gopal AS, et al. Comparison of 2-dimensional and 3-dimensional echocardiography with cineventriculography for measurement of left-ventricular volume in patients. J Am Coll Cardiol 1994;24:1054-63.

15 Gopal AS, Keller AM, Shen ZQ, et al. 3-Dimensional echocardiography-invitro and in-vivo validation of left-ventricular mass and comparison with conventional echocardiographic methods. J Am Coll Cardiol 1994;24:504-13.

16 Gopal AS, Schnellbaecher MJ, Shen ZQ, et al. Freehand three-dimensional echocardiography for determination of left ventricular volume and mass in patients with abnormal ventricles: comparison with magnetic resonance imaging. J Am Soc Echocardiogr 1997; 10:853-61.

17 Gopal AS, Schnellbaecher MJ, Shen ZQ, et al. Freehand three-dimensional echocardiography for measurement of left ventricular mass: in vivo anatomic validation using explanted human hearts. J Am Coll Cardiol 1997;30:802-10. 
18 Pandian NG, Roelandt J, Nanda NC, et al. Dynamic 3-dimensional echocardiography-methods and clinical potential. Echocardiography 1994; 11:237-59

19 Hozumi T, Yoshikawa J, Yoshida K, et al. Three-dimensional echocardiographic measurement of left ventricular volumes and ejection fraction using a multiplane transesophageal probe in patients. Am J Cardiol 1996;78: 1077-80

20 Franke A, Flachskampf FA, Kuhl HP, et al. 3-Dimensional reconstruction of multiplane transesophageal echocardiographic images - report on a new method with clinical examples. Zeitschrift fur Kardiologie 1995;84:633-42.

21 Ludomirsky A, Vermilion R, Nesser J, et al. Transthoracic real-time 3dimensional echocardiography using the rotational scanning approach for data-acquisition. Echocardiography 1994;11:599-606.

22 Fulton DR, Marx GR, Pandian NG, et al. Dynamic three-dimensional echocardiographic imaging of congenital heart defects in infants and children by computer-controlled tomographic parallel slicing using a single integrated ultrasound instrument. Echocardiography 1994;11:155-164.

23 Marx GR, Fulton DR, Pandian NG, et al. Delineation of site, relative size and dynamic geometry of atrial septal-defects by real-time 3-dimensional echocardiography. J Am Coll Cardiol 1995;25:482-90.

24 Salustri A, Spitaels S, McGhie J, et al. Transthoracic 3-dimensional echocardiography in adult patients with congenital heart-disease. J Am Coll Cardiol 1995:26:759-67.

25 Kasprzak JD, Salustri AS, Roelandt JRTC, et al. Three dimensional echocardiography of aortic valve with rotational approach: feasibility, advantages and limitations in comparison with two dimensional echocardiography. Circulation 1996:94:1830.

26 Mele D, Levine RA. Quantitation of ventricular size and function: principles and accuracy of transthoracic rotational scanning. Echocardiography 2000; 17:749-55

27 Kupferwasser I, MohrKahaly S, Stahr P, et al. Transthoracic threedimensional echocardiographic volumetry of distorted left ventricles using rotational scanning. J Am Soc Echocardiogr 1997;10:840-52

28 Acar P, Maunoury C, Antonietti T, et al. Left ventricular ejection fraction in children measured by three-dimensional echocardiography using a new transthoracic integrated 3D-probe-a comparison with equilibrium radionuclide angiography. Eur Heart J 1998;19:1583-8.

29 Nosir YFM, Lequin MH, Vletter WB, et al. Three dimensional echocardiography for quantification of left ventricular volumes and function with comparison to magnetic resonance imaging in both patients and normal subjects. Circulation 1996:94:1233.

30 Yao JF, Cao QL, Masani N, et al. Three-dimensional echocardiographic estimation of infarct mass based on quantification of dysfunctional left ventricular mass. Circulation 1997;96:1660-6

31 Nosir YFM, Lequin MH, Kasprzak JD, et al. Reproducibility and day to day variabilities of three-dimensional echocardiography for left ventricular volumes and ejection fraction calculation with comparison to magnetic resonance imaging. J Am Coll Cardiol 1997;29:7052.

32 Nosir YFM, Vletter WB, vanDomburg RT, et al. Left ventricular ejection fraction in patients with normal and distorted left ventricular geometry: threedimensional echocardiography versus biplane modified Simpson's method with comparison to radionuclide angiography. J Am Coll Cardiol 1997;29:7053

33 Kuhl HP, Franke A, Janssens U, et al. Three-dimensional echocardiographic determination of left ventricular volumes and function by multiplane transesophageal transducer: dynamic in vitro validation and in vivo comparison with angiography and thermodilution. J Am Coll Cardiol 1998;11:1113-24

34 Belohlavek M, Seward JB. Rapid image acquisition and automated determination of left ventricular cavity boundary for precise and accurate volumetry by clinical three-dimensional echocardiography. J Am Coll Cardiol 1997; $29: 7054$

35 Nosir YFM, Vletter WB, Kasprzak JD, et al. Optimal rotational interval for 3-dimensional echocardiography data acquisition for rapid and accurate measurement of left ventricular function. J Am Soc Echocardiogr 2000; 13:715-22.

36 Buck T, Hunold P, Tkalec W, et al. Determination of left ventricular aneurysm surface area by dynamic three-dimensional echocardiography. J Am Coll Cardiol 1997:29:93-117.

37 Teupe C, Takeuchi M, Yao JF, et al. Determination of left ventricular mass by three-dimensional echocardiography: in vitro validation of a novel quantification method using multiple equi-angular rotational planes for rapid measurements. Int J Cardiovasc Imaging 2002;18:161-67.

38 Wang $X$, Zhang $Y$, Zhang W, et al. Quantitative assessment of left ventricular mass by multiplane transesophageal three-dimensional echocardiography. Chinese Med J 1998; 111:475.

39 Kasprzak JD, Vletter WB, Roelandt JRTC, et al. Visualization and quantification of myocardial mass at risk using three-dimensional contrast echocardiography. Cardiovasc Res 1998;40:314-21.
40 Kasprzak JD, Vletter WB, van Meegen JR, et al. Improved quantification of myocardial mass by three-dimensional echocardiography using a deposit contrast agent. Ultrasound in Medicine and Biology 1998;24 647-53.

41 Yao J, Cao WL, Delabays A, et al. Quantification of regional myocardial dysfunction using three-dimensional echocardiography allows accurate estimation of myocardial infarct mass in vivo. Heart 1996;75:62.

42 Leotta D, Munt B, Legget M, et al. Three-dimensional echocardiography by rapid free scanning from multiple transthoracic windows. J Am Coll Cardiol 1997:29:7055.

43 Leotta DF, Munt B, Bolson EL, et al. Quantitative three-dimensional echocardiography by rapid imaging from multiple transthoracic windows: in vitro validation and initial in vivo studies. $J$ Am Soc Echocardiogr 1997; 10:830-9.

44 Kawai J, Tanabe K, Morioka S, et al. Rapid freehand scanning threedimensional echocardiography: accurate measurement of left ventricular volumes and ejection fraction compared with quantitative gated scintigraphy. J Am Soc Echocardiogr 2003;16:110-15.

45 Snyder JE, Kisslo J, von Ramm O. Real-time orthogonal mode scanning of the heart. I. system design. J Am Coll Cardiol 1986;7:1279-85.

46 Sheikh K, Smith SW, von Ramm O, et al. Real-time, three-dimensional echocardiography: feasibility and initial use. Echocardiography 1991;8:119-25.

47 Ota T, Fleishman CE, Li J, et al. Accuracy of manual and semiautomated methods for volume determination by real-time, three dimensional echocardiography. Circulation 1996;94:1235

48 Fleishman CE, Ota T, Ohazama CJ, et al. Real time three-dimensional echocardiography: measurement of left ventricular mass in dogs. Circulation 1996;94:4019

49 Ota T, Jones M, Fleishman CE, et al. Accuracy of left ventricular stroke volume measurement using real-time, three dimensional echocardiography and electromagnetic flow probe in vivo. Circulation 1997:96:1831.

50 Collins M, Hsieh A, Ohazama CJ, et al. Assessment of regional wall motion abnormalities with real-time 3-dimensional echocardiography. J Am Soc Echocardiogr 1999;12:7-14.

51 Shiota T, McCarthy PM, White RD, et al. Initial clinical experience at real-time three-dimensional echocardiography in patients with ischemic and idiopathic dilated cardiomyopathy. Am J Cardiol 1999;84:1068-73.

52 Zwas DR, Takuma S, Mullis-Jansson S, et al. Feasibility of real-time 3dimensional treadmill stress echocardiography. J Am Soc Echocardiogr 1999;12:285-9.

53 Ahmad M, Xie TR, McCulloch M, et al. Real-time three-dimensional dobutamine stress echocardiography in assessment of ischemia: comparison with two-dimensional dobutamine stress echocardiography. J Am Coll Cardiol 2001;37:1303-9.

54 Altmann K Shen ZQ, Boxt $L M$, et al. Comparison of three-dimensional echocardiographic assessment of volume, mass, and function in children with functionally single left ventricles with two-dimensional echocardiography and magnetic resonance imaging. Am J Cardiol 1997;80:1060-5.

55 Teupe C, Takeuchi M, Ram SP, et al. Three-dimensional echocardiography: in vitro validation of a new, voxel-based method for rapid quantification of ventricular volume in normal and aneurysmal left ventricles. Int J Cardiac Imaging 2001;17:99-105

56 Jiang L, Levine RA, Weyman AE. Echocardiographic assessment of right ventricular volume and function. Echocardiography 1997;14:189-205.

57 Vogel M, White PA, Redington AN. In-vitro validation of right-ventricular volume measurement by 3 -dimensional echocardiography. Br Heart $J$ 1995;74:460-3.

58 Vogel M, Gutberlet M, Dittrich S, et al. Comparison of transthoracic three dimensional echocardiography with magnetic resonance imaging in the assessment of right ventricular volume and mass. Heart 1997;78:127-30.

59 Papavassiliou DP, Parks WJ, Hopkins KL, et al. Three-dimensional echocardiographic measurement of right ventricular volume in children with congenital heart disease validated by magnetic resonance imaging. J Am Soc Echocardiogr 1998:11:770-7.

60 Shiota $T$, Jones $M$, Chikada $M$, et al. Real-time three-dimensional echocardiography for determining right ventricular stroke volume in an animal model of chronic right ventricular volume overload. Circulation 1998.97:1897-900.

61 Heusch A, Rubo J, Krogmann ON, et al. Volumetric analysis of the right ventricle in children with congenital heart defects: comparison of biplane angiography and transthoracic 3-dimensional echocardiography. Cardiology in the Young 1999:9:577-84.

62 Bermejo J, Lang RM, Odremen R, et al. 4-D Surface tracking of contrast enhanced transthoracic biplane echocardiograms: a new tool for quantitative assessment of left ventricular function. J Am Coll Cardiol 2003:437A.

63 Sugeng L, Kirkpatrick J, Lang RM, et al. Biplane stress echocardiography using a prototype matrix array transducer allows more rapid acquisition of post-exercise stress images. J Am Soc Echocardiogr (in press). 